\title{
A Tumor-targeting Adenovirus with High Gene-transduction Efficiency for Primary Pancreatic Cancer and Ascites Cells
}

\author{
MASAKI NAGASATO ${ }^{1,2}$, YOSEI RIN ${ }^{1,2}$, YUKI YAMAMOTO ${ }^{1,2}$, MARINA HENMI $^{1,2}$, \\ NOBUYOSHI HIRAOKA ${ }^{3}$, FUMIKO CHIWAKI ${ }^{4}$, KEISUKE MATSUSAKI ${ }^{5}$, \\ MASATOSHI TAGAWA ${ }^{6}$, HIROKI SASAKI ${ }^{4}$ and KAZUNORI AOKI ${ }^{1}$ \\ ${ }^{1}$ Division of Molecular and Cellular Medicine, ${ }^{3}$ Division of Molecular Pathology, and \\ ${ }^{4}$ Department of Translational Oncology, National Cancer Center Research Institute, Tokyo, Japan; \\ ${ }^{2}$ NCC Cancer Science, Tokyo Medical and Dental University, Tokyo, Japan; \\ ${ }^{5}$ Kaname Second Clinic, Kanamecho Hospital, Tokyo, Japan; \\ ${ }^{6}$ Division of Pathology and Cell Therapy, Chiba Cancer Center Research Institute, Chiba, Japan
}

\begin{abstract}
Background: Optimizing targeting strategies for vectors in order to enhance antitumor activity and secure patient safety is important for cancer gene therapy. We previously identified two pancreatic cancer-targeting ligands (PFWSGAV: PFW and SYENFSA: SYE) by screening an adenovirus library in vivo and in vitro, respectively. Materials and Methods: To examine clinical usefulness, we assessed gene-transduction efficiency using surgicallyresected pancreatic cancer specimens and ascites cells. Results: For surgical specimens, vectors displaying PFW and SYE improved transduction efficiency by 4.4- and 4.3fold, respectively. The SYE-displaying vector was $>2$-fold more efficient for all seven cases, whereas the PFWdisplaying vector increased efficiency in two out of four cases. For ascites samples, both vectors increased genetransduction efficiency of epithelial cell adhesion molecule (EpCAM)-positive ascites cells by $>2$-fold in two out of five cases. Conclusion: Both vectors enhanced adenovirus infectivity of pancreatic cancer cells and have potential for gene therapy of pancreatic cancer; therefore they should be further evaluated in clinical studies.
\end{abstract}

Pancreatic cancer is the fourth leading cause of cancerrelated death in Japan as well as in Western countries. The 5 -year survival rate for patients with pancreatic cancer is less than $5 \%$, and overcoming this poor prognosis remains one of the most formidable challenges in oncology $(1,2)$.

Correspondence to: Kazunori Aoki, Division of Molecular and Cellular Medicine, National Cancer Center Research Institute, 5-11 Tsukiji, Chuo-ku, Tokyo 104-0045, Japan. Tel: +81 335422511 (Ext. 4400). Fax: +81 332481631, e-mail: kaoki@ncc.go.jp

Key Words: Adenovirus delivery, gene therapy, pancreatic cancer.
Surgical resection is the only curative treatment option but the 5-year survival rate for patients who undergo this procedure is only $15-20 \%$. Advances in neoadjuvant and adjuvant chemotherapeutic regimens have resulted in some improvement in outcome (2-4). Existing systemic therapies are only modestly effective and the median survival for patients with metastatic disease remains 6 months $(2,3,5)$.

Common manifestations of end-stage pancreatic cancer include gastric outlet obstruction, deep venous thrombosis, cachexia, anasarca, and ascites (6). Ascites develops in $20 \%$ of patients with pancreatic cancer, which causes deteriorated quality of life; the duration of survival after ascites development is only a couple of months. Available medical treatments for patients with malignant ascites include the use of diuretics or paracentesis $(7,8)$. A novel approach to treat peritoneal dissemination is thus needed.

Recombinant adenovirus vectors have been widely used for gene delivery and have been employed with several gene therapy approaches (9-11). However, the lack of infection specificity due to the widespread distribution of primary cellular adenovirus receptors has prevented in vivo cancerspecific gene transduction $(9,11,12)$. Most currently-used adenovirus vectors are based on serotype 5 (Ad5), which requires two distinct and sequential steps for entry into susceptible cells. The initial step is an interaction between the fiber protein and its cellular receptor, coxsackievirus and adenovirus receptor (CAR) $(10,11)$. The next step, internalization of the virus, is promoted by the interaction between the penton base and $\alpha \mathrm{v}$ integrins on the cell surface $(10,11)$. Retargeting has been achieved through direct genetic modifications of the capsid proteins; targeting ligands that are deficient for native tropism can be incorporated into the C-terminal and HI-loop of fiber proteins $(10,11)$. However, redirecting adenovirus vectors by engineering the capsid-coding region has shown limited 
success because proper targeting ligands are generally unknown. In order to overcome this limitation, we have developed a system for producing adenoviral libraries, wherein a variety of peptides are displayed on the HI-loop of the fiber knob; screening such peptides has led to the successful selection of several adenoviral vectors that exhibit high infectivity of target cells (13-17).

SYENFSA (SYE) is one sequence that was selected from the library through in vitro screening using the AsPC-1 pancreatic cancer cell line, and this SYE-displaying adenovirus vector resulted in a higher gene-transduction efficiency in four out of five pancreatic cancer cell lines and five out of six surgical resection specimens $(17,18)$. The PFWSGAV (PFW) sequence was identified from the library through in vivo screening using a murine model of peritoneal dissemination of AsPC-1 cells; the PFW-displaying adenovirus vector showed high infectivity compared to that of a non-targeting vector when tested with AsPC-1 peritoneal tumors, but not with other peritoneal tumors and organs (16). When we compared the infectivity of both targeting vectors, the gene-transduction efficiency of the SYE-displaying vector was higher than that of the $\mathrm{PFW}$-displaying vector when using cultured AsPC-1 cells, whereas the PFWdisplaying vector showed significantly higher infectivity than the SYE-displaying vector when using AsPC-1 peritoneal tumors (16). In this study, in order to examine the usefulness of vectors in a clinical setting, we compared the efficiency of both vectors using clinical samples namely surgical specimens and ascites cells from cases of human pancreatic cancer.

\section{Materials and Methods}

Recombinant adenovirus vectors. Adenovirus vectors were constructed as previously described (15-17). Briefly, the adenovirus vectors included four point mutations in the AB-loop of the fiber knob, which reduces CAR binding. Ad $\triangle$ CAR-LucEGFP-SYE and Ad $\triangle C A R-S Y E$ encode the SYENFSA sequence, and Ad $\triangle C A R-$ LucEGFP-PFW encodes the PFWSGAV sequence in the HI-loop of the fiber knob. The E1 gene of Ad $\Delta$ CAR-LucEGFP, Ad $\Delta$ CARLucEGFP-SYE, and Ad $\triangle$ CAR-LucEGFP-PFW was replaced with the cytomegalovirus (CMV) immediate early promoter-driven luciferase-enhanced green fluorescent protein (EGFP) fusion gene (LucEGFP). In Ad $\triangle \mathrm{CAR}$ and Ad $\triangle \mathrm{CAR}-\mathrm{SYE}$, the E3 region was replaced with the CMV promoter-driven EGFP gene (Figure 1A). The adenovirus vectors were expanded in 293-38 cells (13). The physical particle concentration [viral particles $(\mathrm{vp}) / \mathrm{ml}$ of virus preparation] was determined by optical absorbance (OD260) measurements.

Surgical resection specimens and ascites cells from human pancreatic cancer. Surgical specimens and ascites cells from cases of human pancreatic cancer were obtained according to the Declaration of Helsinki Principles and the guideline of the Ethics Committee of the National Cancer Center (Tokyo, Japan) and Kanamecho Hospital (Tokyo, Japan). Surgical tissues were processed into small pieces of tissues or single cells, and were cultured in RPMI-1640 medium (Nissui Pharmaceutical, Tokyo, Japan) with $10 \%$ fetal bovine serum (FBS). Ascites cells from patients with pancreatic cancer were cultured in RPMI-1640 medium with $10 \%$ FBS

Luciferase activity assay. In order to examine the gene-transduction efficiency of targeting adenovirus vectors, small pieces of tissue derived from surgical specimens were infected with adenoviruses at $1 \times 10^{9}$ vp per well of a 24 -well plate. Twenty-four hours after infection, the cells were lysed with $100 \mu$ of lysis buffer (Reporter Lysis Buffer; Promega, Madison, WI, USA) and then $100 \mu \mathrm{l}$ of assay substrate (PicaGene; TOYO B-Net Co., LTD, Tokyo, Japan) was added. The light units of luciferase activity were measured using a luminometer (Mini Lumat LB9506; Berthold GmbH \& Co. KG, Bad Wildbad, Germany).

Flow cytometry. Flow cytometry was performed to assess the percentage of $\mathrm{EGFP}^{+}$cells. Single cells were prepared from surgically resected tissues, and were filtered using a Falcon $40-\mu \mathrm{m}$ Cell Strainer (Corning, New York, NY, USA). The cells were then infected with viruses at $1 \times 10^{3} \mathrm{vp} / \mathrm{cell}$, and were harvested $24-48 \mathrm{~h}$ after viral infection. Subsequently, the cells were stained using LIVE/DEAD cell viability assay kit (Thermo Fisher Scientific, Waltham, MA, USA) for the selection of live cells, which was followed by fixation using $4 \%$ paraformaldehyde (Wako Pure Chemical Industries, Ltd., Tokyo, Japan) for $20 \mathrm{~min}$ at room temperature.

Ascites cells were infected with viruses at $3 \times 10^{3} \mathrm{vp} / \mathrm{cell}$, and were harvested $24-48 \mathrm{~h}$ after viral infection. The cells were stained using LIVE/DEAD, which was followed by fixation with $4 \%$ paraformaldehyde. Subsequently, the cells were stained with phycoerythrin (PE)-conjugated monoclonal antibody to CD326 epithelial cell adhesion molecule (EpCAM) (HEA-125; Miltenyi Biotec, Bergisch Gladbach, Germany) or PE-conjugated mouse IgG isotype control (MOPC-21; BioLegend, San Diego, CA, USA) for $30 \mathrm{~min}$ on ice, and analyzed using an LSRFortessa X-20 Cell Analyzer (BD Biosciences, San Jose, CA, USA).

Statistical analysis. Comparative analysis of luciferase activity was performed using Student's $t$-test; differences were considered statistically significant when the $p$-value was less than 0.05 .

\section{Results}

Infectivity of adenovirus vectors using tissue derived from surgical specimens. Firstly, in order to compare the structure of the SYE ligand-displaying fiber knob to that of the PFW ligand-displaying fiber knob, three-dimensional models were constructed using SWISS-MODEL (http://swissmodel. expasy.org/) based on homology modeling. The structure of the SYE-displaying fiber knob was entirely different from that of the PFW-displaying fiber knob (Figure 1B).

Subsequently, in order to compare the gene-transduction efficiency of adenovirus vectors displaying the selected peptides (PFWSGAV and SYENFSA), small pieces of tissues derived from surgical specimens were infected with Ad $\triangle$ CAR-LucEGFP-PFW and Ad $\Delta$ CAR-LucEGFP-SYE. 
A Luciferase-EGFP (in deleted E1)

Ad $\triangle$ CAR-LucEGFP

\section{CAR binding ablation}

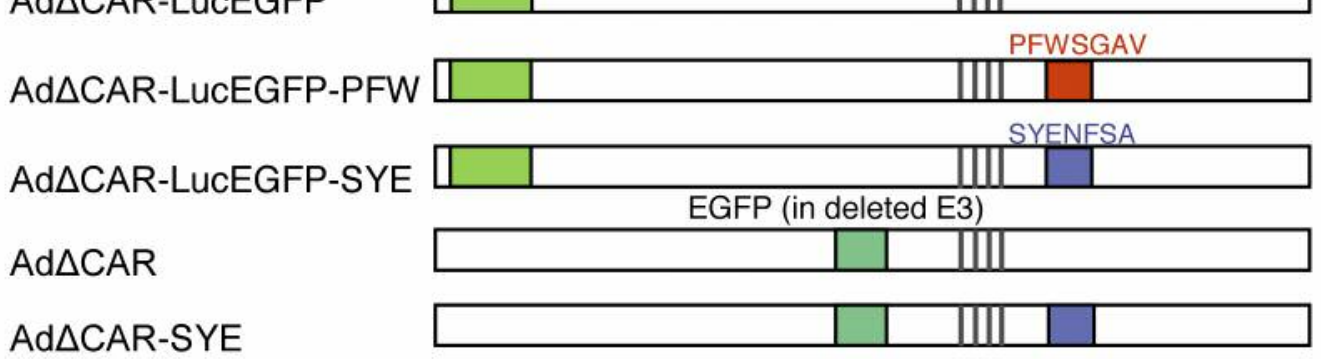

B
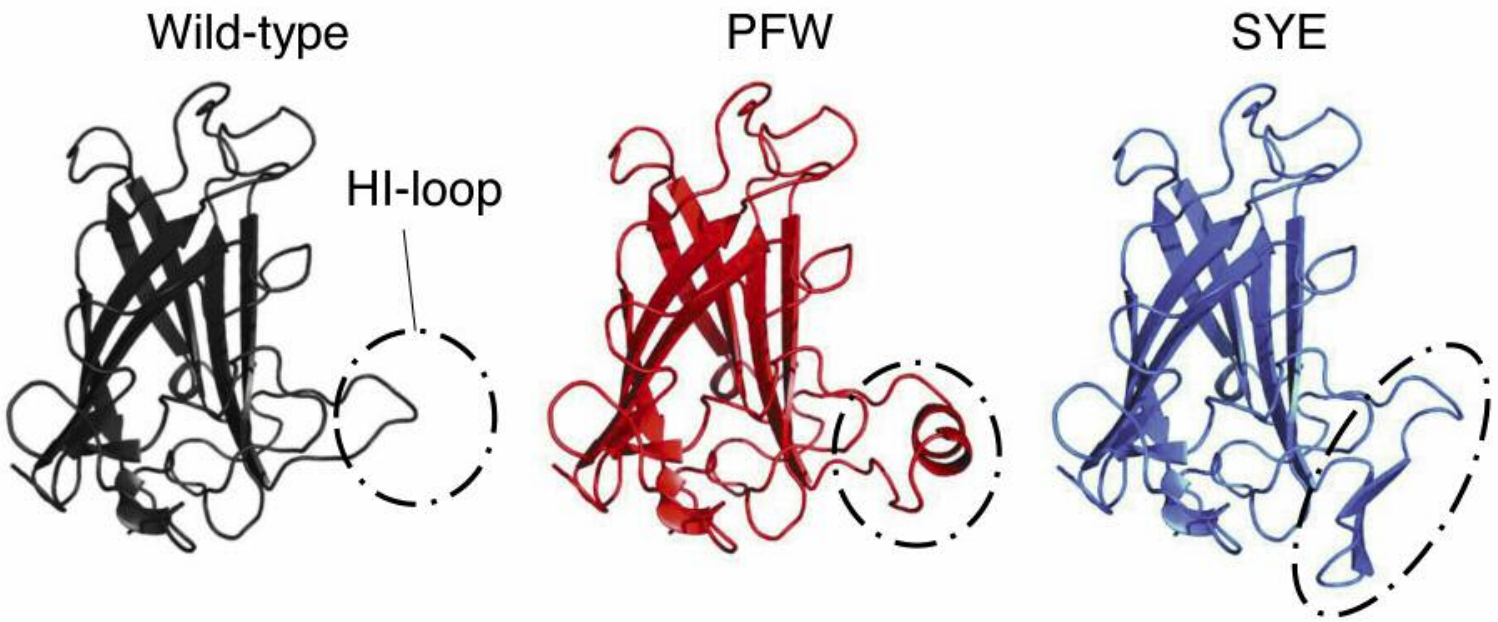

Figure 1. Structure of adenovirus constructs displaying pancreatic cancer-targeting ligands. A: Design of adenovirus constructs. Four-pointmutations were inserted into the region encoding the AB-loop to reduce Coxsackievirus and adenovirus receptor (CAR) binding. The E1 gene was replaced with the cytomegalovirus $(C M V)$ immediate early promoter-driven luciferase-enhanced green fluorescent protein (EGFP) fusion gene (LucEGFP)

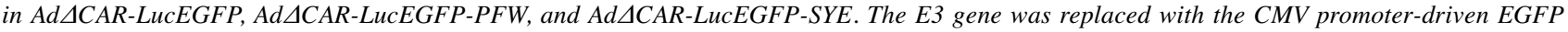
gene in Ad $\triangle C A R$ and Ad $\triangle C A R-S Y E$. Ad $\triangle C A R$-LucEGFP-PFW displays the pancreatic cancer targeting sequence PFW on the fiber knob. Ad $\triangle C A R$ LucEGFP-SYE and AdDCAR-SYE display the pancreatic cancer targeting sequence SYE on the fiber knob. B: Three-dimensional protein structures of the adenovirus fiber knob predicted by SWISS-MODEL (University of Basel, Basel, Switzerland) using PyMOL.

Twenty-four hours after infection, $\mathrm{EGFP}^{+}$cells were detected by fluorescence microscopy in the tissues infected with Ad $\triangle$ CAR-LucEGFP-PFW and Ad $\triangle$ CAR-LucEGFP-SYE, whereas $\mathrm{EGFP}^{+}$cells were not detected in normal pancreas (Figure 2A). Luciferase assays also showed that both vectors resulted in a high gene-transduction efficiency (at the same level) in pancreatic cancer, whereas the activity was not enhanced in normal pancreatic tissue when compared to that after mock infection (Figure 2B).

Infectivity of adenovirus vectors using single cells derived from surgical specimens. In order to confirm the enhanced genetransduction efficiency of adenovirus vectors displaying selected peptides when compared to that of non-targeting control vectors in human pancreatic cancer, single cells were prepared from surgical specimens and infected with Ad $\triangle$ CAR-LucEGFPPFW, Ad $\triangle$ CAR-LucEGFP, Ad $\triangle$ CAR-SYE, and Ad $\triangle$ CAR. Subsequently, the percentage of EGFP ${ }^{+}$cells was assessed by flow cytometry $24 \mathrm{~h}$ after infection. Ad $\Delta$ CAR-LucEGFP-PFW resulted in a 4.4-fold higher frequency of $\mathrm{EGFP}^{+}$cells in pancreatic cancer, compared to that when using a non-targeting vector, whereas $\mathrm{EGFP}^{+}$cells were not detected in normal pancreas (Figure 2C, left panel). Similarly, the frequency of $\mathrm{EGFP}^{+}$cells infected with Ad $\triangle \mathrm{CAR}-\mathrm{SYE}$ in pancreatic cancer was 4.2-fold higher, and EGFP expression was not recognized in normal pancreas (Figure 2C, right panel). 


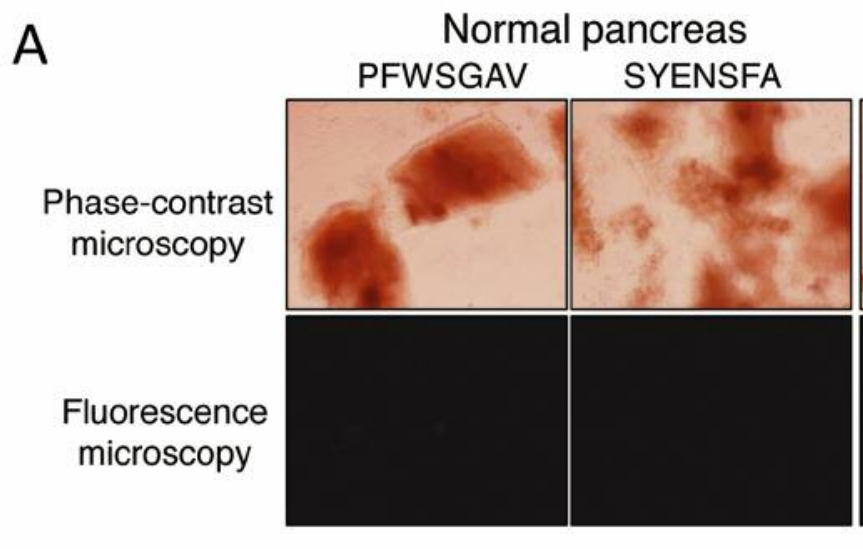

B

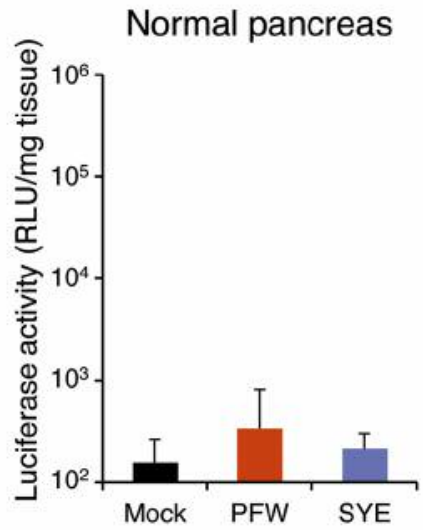

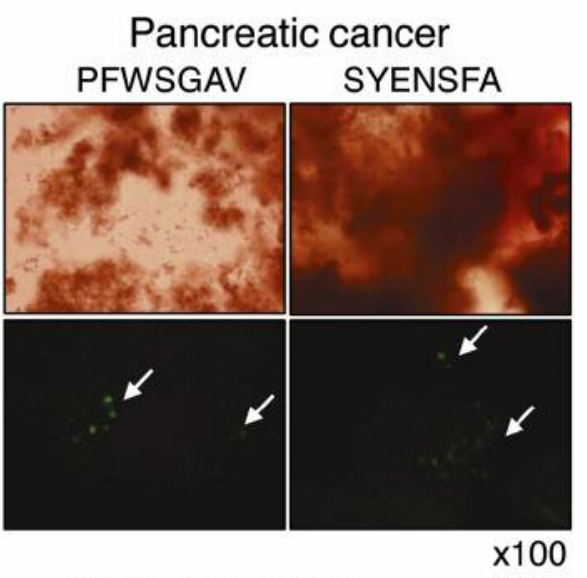

Pancreatic cancer
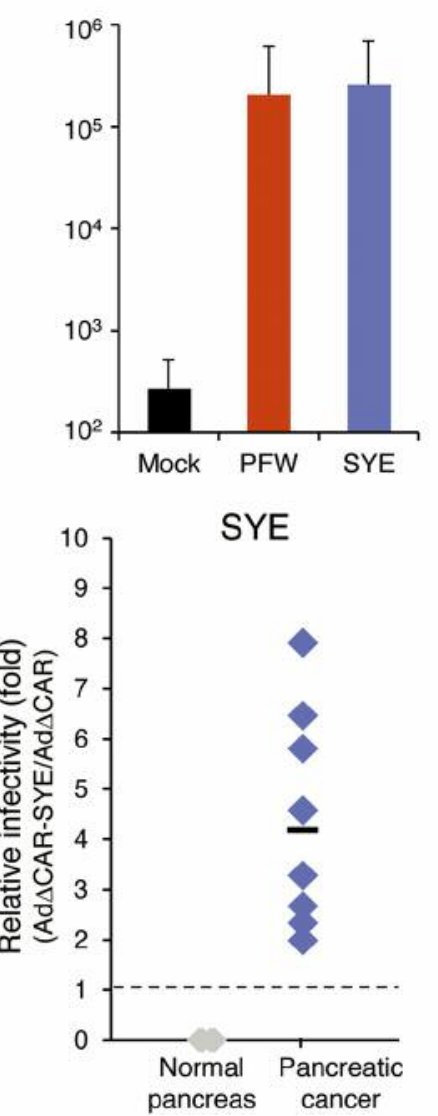

Figure 2. Gene-transduction efficiency of adenovirus vectors displaying pancreatic cancer-targeting ligands using surgical specimens of human pancreatic cancer. A: Photographs of pancreatic cancer tissues infected with viruses. Small pieces of tissues derived from surgical specimens were infected with adenovirus vectors (Ad $\triangle C A R$-LucEGFP-PFW and Ad $\triangle C A R$-LucEGFP-SYE). Arrows: Cells positive for enhanced green fluorescent protein (EGFP). B: Luciferase activity of tissues infected with viruses. Small pieces of human pancreatic cancer ( $n=11)$ and human normal pancreas $(n=5)$ tissues were infected with AdDCAR-LucEGFP-PFW and AdACAR-LucEGFP-SYE. Twenty-four hours after infection, luciferase activity was measured. The graph depicts the luciferase activity per mg of tumor tissue. RLU: Relative light unit. Differences were not significant. C: Relative infectivity of pancreatic cancer tissues infected with targeting viruses compared to that using the non-targeting virus. Single cells were prepared from surgical specimens of human pancreatic cancer, and infected with viruses at $1 \times 10^{3}$ viral particles/cell. Left panel: Human normal pancreatic cells $(n=1)$ and human pancreatic cancer cells $(n=4)$ were infected with adenovirus vectors (Ad $\triangle C A R$-LucEGFP-PFW and AdDCAR-LucEGFP), and $24 \mathrm{~h}$ later, $E G F P^{+}$cells were detected by flow cytometry. The relative frequency of EGFP+ cells is presented as the percentage of $E G F P^{+}$cells infected with AdDCAR-LucEGFP-PFW relative to that with AdACAR-LucEGFP. Right panel: Human normal pancreatic cells ( $n=4)$ and human pancreatic cancer cells $(n=9)$ were infected with adenovirus vectors (AdDCAR-SYE and AdACAR). The relative frequency of EGFP+ cells is

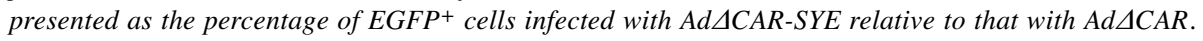


A
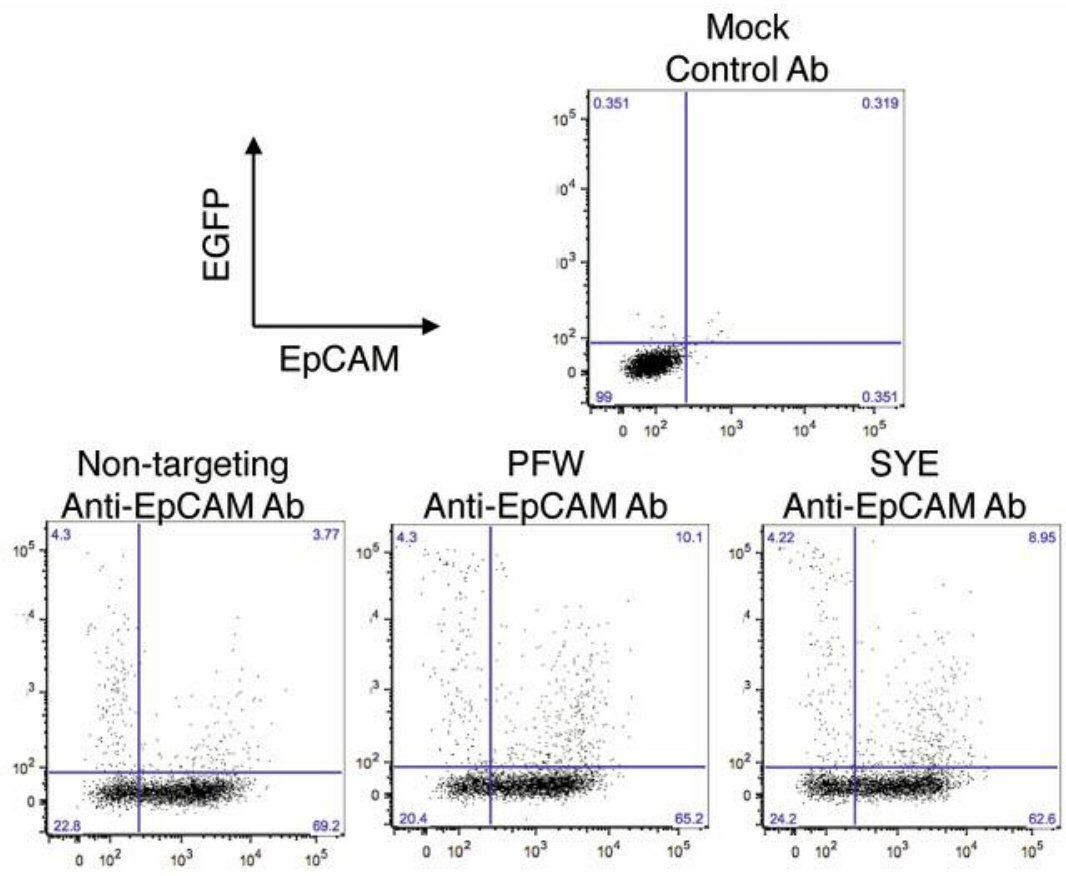

B
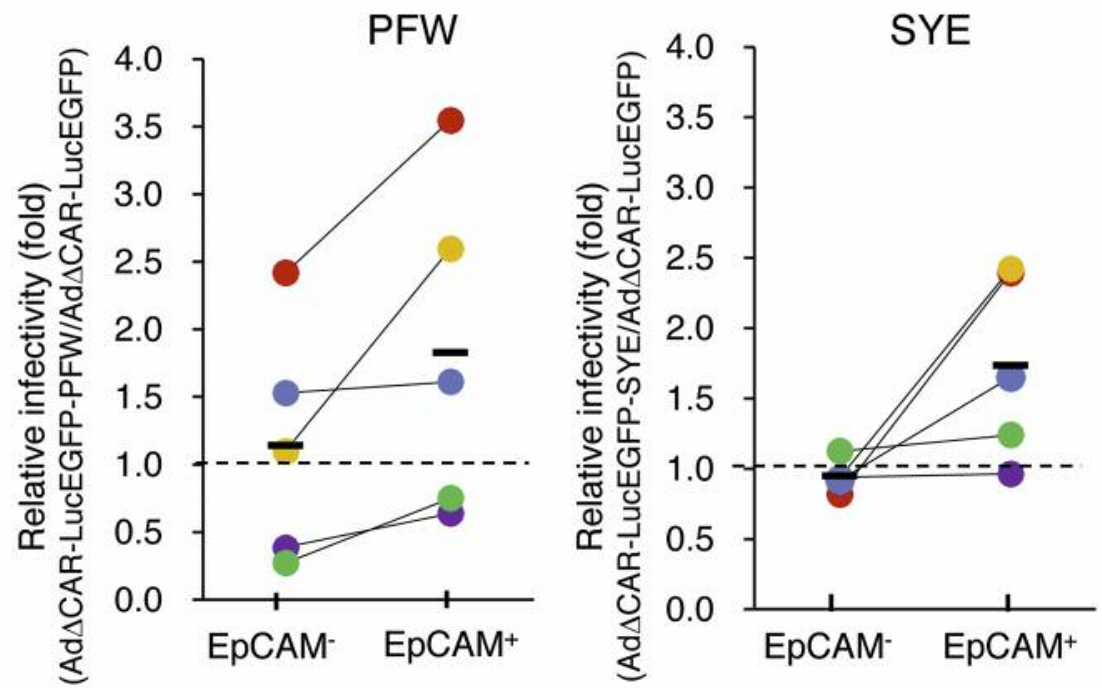

Figure 3. Gene-transduction efficiency of adenovirus vectors displaying pancreatic cancer-targeting peptides using ascites cells of human pancreatic cancer. A: Representative plot of flow cytometric analysis. Pancreatic ascites cells $(n=5)$ were infected with Ad $\triangle C A R-L u c E G F P, A d \triangle C A R-L u c E G F P$ $P F W$, and AdDCAR-LucEGFP-SYE at $1 \times 10^{3}$ viral particles/cell, and $24 \mathrm{~h}$ later, the surface of cells was stained using an antibody to epithelial cell adhesion molecule (EPCAM). Mock-infected ascites cells were stained using isotype control antibody. B: Cells positive for enhanced green fluorescent protein (EGFP) cells in fractions of EpCAM+ and EpCAM- cells infected with viruses. The relative frequency of EGFP+ cells is presented as the percentage of EGFP ${ }^{+}$cells infected with AdACAR-LucEGFP-PFW or AdDCAR-LucEGFP-SYE relative to that with AdDCAR-LucEGFP.

The average relative infectivity of the PFW-displaying vector (Ad $\triangle$ CAR-LucEGFP-PFW) was similar to that of the SYE-displaying vector (Ad $\Delta$ CAR-SYE). However, the SYEdisplaying vector resulted in $>2$-fold higher gene- transduction efficiency in all seven cases, whereas the PFWdisplaying vector increased efficiency in two out of four cases (Figure 2C), suggesting that the SYE ligand is able to target a wide range of pancreatic cancer cells. 
Infectivity of adenovirus vectors using ascites cells. For comparison of the gene-transduction efficiencies of SYEand PFW-displaying vectors for pancreatic peritoneal metastasis, ascites cells, instead of peritoneal tumors, were collected from five patients with pancreatic cancer, and these cells were infected with Ad $\triangle$ CAR-LucEGFP-PFW and Ad $\triangle$ CAR-LucEGFP-SYE. The percentage of EGFP ${ }^{+}$ cells in the $\mathrm{EpCAM}^{+}$(a carcinoma marker) and $\mathrm{EpCAM}^{-}$ cell fractions was assessed by flow cytometry. The efficiencies of Ad $\Delta$ CAR-LucEGFP-PFW and Ad $\Delta$ CARLucEGFP-SYE were $>2$-fold higher, when compared to that using a non-targeting vector in two out of five cases (Figure $3 \mathrm{~A}$ and $\mathrm{B}$ ).

Ad $\triangle$ CAR-LucEGFP-PFW resulted in $>3$-fold higher genetransduction efficiency of $\mathrm{EpCAM}^{+}$cells for one case; however, the efficiency was also increased in $\mathrm{EpCAM}^{-}$cells from the same patient (Figure 3B, left). In contrast, Ad $\triangle$ CAR-LucEGFP-SYE resulted in a higher genetransduction efficiency only in $\mathrm{EpCAM}^{+}$cells, and not in $\mathrm{EpCAM}^{-}$cells, indicating the pancreatic cancer-specificity of the SYE ligand (Figure 3B, right). These results suggest that the SYE-displaying vector is more specific for pancreatic cancer ascites cells than the PFW-displaying vector.

\section{Discussion}

We previously reported that sequences selected from the same adenovirus library, through in vivo and in vitro screening using AsPC-1 pancreatic cancer cells, were completely different $(16,17)$. In this study, we compared the gene-transduction efficiency of both targeting vectors using clinical pancreatic cancer samples as a pre-clinical study. In surgical specimens, the SYE-displaying adenovirus vector resulted in higher infectivity in all cases, whereas the PFWdisplaying adenovirus vector showed higher infectivity in two out of four cases. In ascites cells, both targeting vectors showed high infectivity in two out of five cases. These results suggest that both vectors have potential for future clinical applications; in addition, the SYE-displaying vector seems to have higher specificity and target a wider population of pancreatic cancer cells than the PFWdisplaying vector.

The infectivity of targeting vectors was different between primary lesions and ascites cells. The expression levels and types of cell surface receptors might be different in primary and metastatic lesions, which could be affected by various factors such as cytokines and growth factors produced by the tumor, stromal, and immune cells in the tumor microenvironment. Therefore, identification of the receptor for PFW and SYE peptides is useful for understanding the molecular characteristics of their target cells and this could be applied for diagnostic tests such as the detection of disease relapse (11).
Both targeting vectors resulted in high gene-transduction efficiency in a significant population of primary lesion and ascites cells. The direct injection of pancreatic cancertargeting adenovirus vectors including oncolytic viruses is a promising therapeutic strategy for locally advanced pancreatic cancer, in order to control local lesions and to prevent distant metastasis $(11,18)$. In contrast, many patients with advanced-stage disease suffer from the accumulation of ascites via peritoneal dissemination of the primary tumor (8). Treatment with peritoneal tumor-targeting vectors immediately after surgery could delay the occurrence of metastases and prolong patient survival. In addition, suppressing peritoneal tumors with targeting vectors should improve patient quality of life (8).

One of the most important issues for achieving success in clinical trials is the selection of patients with tumor tissues that are suitable for this targeting strategy. The selection of patients based on high viral infectivity of biopsy samples might be feasible in clinical settings. If SYE- and PFW-displaying adenovirus vectors are clinically available, a suitable virus for each patient could be selected based on viral infectivity of biopsy samples and patient-derived xenografts (11).

In conclusion, pancreatic cancer-targeting adenovirus vectors displaying PFW and SYE showed a high genetransduction efficiency using clinical pancreatic cancer samples. Thus, both vectors have potential for the treatment for pancreatic cancer.

\section{Conflicts of Interest}

The Authors have no conflict of interest in regard to this study.

\section{Acknowledgements}

This work was supported in part by grants-in-aid for research from the Japan Agency for Medical Research and Development, and by JSPS KAKENHI (JP16K08752), and by National Cancer Center Research and Development Fund (29-A-2 and 29-A-7). We also acknowledge the technical assistance of Ms. S. Mito and Mrs. K. Onobori.

\section{References}

1 Siegel RL, Miller KD and Jemal A: Cancer Statistics, 2017. CA Cancer J Clin 67: 7-30, 2017.

2 Chadha AS, Khoo A, Aliru ML, Arora HK, Gunther JR and Krishnan S: Recent advances and prospects for multimodality therapy in pancreatic cancer. Semin Radiation Oncol 26: 320337, 2016.

3 Werner J, Combs SE, Springfeld C, Hartwig W, Hackert T and Buchler MW: Advanced-stage pancreatic cancer: therapy options. Nat Rev Clin Oncol 10: 323-333, 2013.

4 Valsecchi ME, Diaz-Canton E, de la Vega M and Littman SJ: Recent treatment advances and novel therapies in pancreas cancer: A review. J Gastrointest Cancer 45: 190-201, 2014. 
5 Mosquera C, Maglic D and Zervos EE: Molecular targeted therapy for pancreatic adenocarcinoma: A review of completed and ongoing late phase clinical trials. Cancer Genetics 209: 567$581,2016$.

6 Adam RA and Adam YG: Malignant ascites: past, present, and future. J Am Coll Surg 198: 999-1011, 2004.

7 Zervos EE, Osborne D, Boe BA, Luzardo G, Goldin SB and Rosemurgy AS: Prognostic significance of new onset ascites in patients with pancreatic cancer. World J Surg Oncol 4: 16, 2006.

8 Hicks AM, Chou J, Capanu M, Lowery MA, Yu KH and O'Reilly EM: Pancreas adenocarcinoma: Ascites, clinical manifestations, and management implications. Clin Colorectal Cancer 15: 360-368, 2016

9 Pesonen S, Kangasniemi L and Hemminki A: Oncolytic adenoviruses for the treatment of human cancer: focus on translational and clinical data. Mol Pharm 8: 12-28, 2011.

10 Beatty MS and Curiel DT: Chapter two-Adenovirus strategies for tissue-specific targeting. Adv Cancer Res 115: 39-67, 2012.

11 Yamamoto Y, Nagasato M, Yoshida T and Aoki K: Recent advances in genetic modification of adenovirus vectors for cancer treatment. Cancer Sci, 2017. doi: 10.1111/cas.13228 [Epub ahead of print]

12 Yamamoto $M$ and Curiel DT: Current issues and future directions of oncolytic adenoviruses. Mol Ther 18: 243-250, 2010.

13 Hatanaka K, Ohnami S, Yoshida K, Aoyagi K, Sasaki H, Asaka M, Terada M, Yoshida T and Aoki K: A simple and efficient method for constructing an adenoviral cDNA expression library. Mol Ther 8: 158-166, 2003.
14 Yamamoto Y, Goto N, Miura K, Narumi K, Ohnami S, Uchida H, Miura Y, Yamamoto M and Aoki K: Development of a novel efficient method to construct an adenovirus library displaying random peptides on the fiber knob. Mol Pharm 11: 1069-1074, 2014.

15 Miura Y, Yoshida K, Nishimoto T, Hatanaka K, Ohnami S, Asaka M, Douglas JT, Curiel DT, Yoshida T and Aoki K: Direct selection of targeted adenovirus vectors by random peptide display on the fiber knob. Gene Ther 14: 1448-1460, 2007.

16 Nishimoto T, Yamamoto Y, Yoshida K, Goto N, Ohnami S and Aoki K: Development of peritoneal tumor-targeting vector by in vivo screening with a random peptide-displaying adenovirus library. PLoS One 7: e45550, 2012.

17 Nishimoto T, Yoshida K, Miura Y, Kobayashi A, Hara H, Ohnami S, Kurisu K, Yoshida T and Aoki K: Oncolytic virus therapy for pancreatic cancer using the adenovirus library displaying random peptides on the fiber knob. Gene Ther 16 : 669-680, 2009.

18 Yamamoto Y, Hiraoka N, Goto N, Rin Y, Miura K, Narumi K, Uchida $\mathrm{H}$, Tagawa $\mathrm{M}$ and Aoki $\mathrm{K}$ : A targeting ligand enhances infectivity and cytotoxicity of an oncolytic adenovirus in human pancreatic cancer tissues. J Control Release 192: 284-293, 2014.

Received May 3, 2017

Revised May 16, 2017

Accepted May 19, 2017 